\title{
Avaliação da disponibilidade, adsorção e níveis críticos de fósforo em diferentes solos ${ }^{1}$
}

\author{
Fábio Broggi ${ }^{2}$ Fernando José Freire ${ }^{3}$, Maria Betânia Galvão dos Santos Freire ${ }^{3}$, Clístenes Williams Araújo do \\ Nascimento $^{3}$, Alexandre Campelo de Oliveira ${ }^{4}$
}

\section{RESUMO}

A avaliação da disponibilidade de $\mathrm{P}$ em solos mineralogicamente diferentes por um único método de análise pode ocasionar estimativas inadequadas dessa disponibilidade. O objetivo desta pesquisa foi extrair P por diferentes extratores químicos em solo com elevado teor de Ca, comparativamente a solo hematítico, e estudar as relações entre os níveis críticos e a adsorção de P pelo tempo de incubação dos solos. Assim, realizou-se um experimento utilizando amostras subsuperficiais de um Vertissolo Ebânico órtico (VEo), textura argilosa, com predomínio de argilominerais 2:1 rico em Ca e de um Nitossolo Vermelho distroférrico (NVdf), textura muito argilosa, predominantemente hematítico, coletados no Sertão e na Zona da Mata do Estado de Pernambuco, respectivamente. As doses de P foram definidas em função dos níveis 0; 0,2; 0,4; 0,8; e 1,0 da capacidade máxima de adsorção de P (CMAP) de cada solo e incubadas por 90, 60, 30, 15 e 0,5 dias. O experimento foi realizado em casa de vegetação, em esquema fatorial (dois solos, cinco doses de P e cinco tempos de incubação) em delineamento de blocos casualizados, com três repetições. O P disponível foi determinado pelos extratores Mehlich-1, Mehlich-3 e Bray-1 e utilizou-se o milho como planta indicadora. A capacidade de predição do P disponível do Mehlich-1 não foi adequada para o solo com predomínio de argilominerais 2:1 rico em Ca. Para este caso, a maior operacionalidade do Bray-1 credencia esse extrator como mais recomendado. Os níveis críticos no solo apresentaram-se decrescentes com o aumento do tempo de incubação, independentemente do solo.

Palavras chave: P lábil, extratores de P, capacidade máxima de adsorção de $\mathrm{P}$

\section{ABSTRACT}

\section{Evaluation of availability, adsorption and $P$ critical levels in different soils}

The evaluation of $\mathrm{P}$ availability in soils with different mineralogy using a same methodology can produce inappropriate estimates. The aim of this work was to evaluate P availability using different chemical extractors in soil with high Ca concentration, in comparison with hemathitic soil, and to study the relations between the critical levels and the $\mathrm{P}$ adsorption as a function of the soil incubation time. An experiment using subsurface samples of a Orthic Ebanic Vertisol (VEo) (Mollisol), clay textured with predominance of 2:1 clay mineral rich in Ca and a Distroferric Red Nitosol (NVdf) (Nitosol), high clay content, predominantly hematitic, colleted at the semi-arid and humid regions of Pernambuco State, Brazil, respectively. The P doses were defined as a function of the levels 0, 0.2, 0.4, 0.8 and 1.0 of the Maximum Capacity of P Adsorption (MCPA) of each soil and incubated for 90, 60, 30, 15 and 0.5 days. The experiment was carried out in a greenhouse in a factorial scheme (2 soils, 5 P doses and 5 different periods of incubation), in a

Recebido para publicação em novembro de 2008 e aprovado em março de 2010

'Parte da dissertação de mestrado apresentada pelo primeiro autor à Universidade Federal Rural de Pernambuco (UFRPE).

2Engenheiro-Agrônomo, Mestre. Departamento de Agronomia, UFRPE, Rua Dom Manoel de Medeiros, s/n, 52171-900, Recife, Pernambuco, Brasil. fabiobroggi@bol.com.br. ${ }^{3}$ Engenheiros-Agrônomos, Doutores. Departamento de Agronomia, UfRPE, Rua Dom Manoel de Medeiros, s/n, 52171-900, Recife, Pernambuco, Brasil. f.freire@depa.ufrpe.br, betânia@depa.ufrpe.br, clistenes@depa.ufrpe.br.

${ }^{4}$ Engenheiro-Agrônomo, Doutorando. Departamento de Agronomia, UFRPE, Rua Dom Manoel de Medeiros, s/n, 52171-900, Recife, Pernambuco, Brasil. alexcampelo@oi.com.br. 
randomized blocks design, with three replicates each. The available P was determined by the extractors Mehlich-1, Mehlich-3 and Bray-1 and corn was used as indicative plant. The capacity of prediction of available P by Mehlich-1 was not suitable for the soil with predominance of 2:1 clay mineral rich in Ca. For this special case, the highest operationallity of Bray-1 accredits this extractor as the most recommended. The critical levels in the soil decreased with the increase in incubation time, independently of the soil.

Key words: $\mathrm{P}$ labile, $\mathrm{P}$ extractors, maximum capacity of $\mathrm{P}$ adsorption.

\section{INTRODUÇÃO}

A disponibilidade de $\mathrm{P}$ às plantas tem sido avaliada por numerosas técnicas de extração, o que, de certa forma, é um indicativo da complexidade do comportamento do elemento no solo, assim como da falta de concordância sobre qual seria o método mais adequado (Silva \& Raij, 1999). A deficiência de P nos solos tropicais é intensificada devido ao $\mathrm{pH}$ ácido e à presença de óxidos cristalinos e amorfos de Fe e Al. Tais minerais apresentam grupamentos superficiais $\mathrm{Fe}-\mathrm{OH}$ e $\mathrm{Al}-\mathrm{OH}$, nos quais o fosfato pode ser adsorvido por meio de troca de ligantes com o estabelecimento de ligações covalentes. Além disso, sua forma estrutural em adição a sua superfície específica influencia na adsorção de fosfato pelos solos (Mesquita Filho \& Torrent, 1993).

Nos estados do Nordeste os solos são, em sua grande maioria, menos ácidos do que nas outras regiões do País, sendo, também, comum a formação de compostos pouco solúveis de fosfatos de cálcio. Nessas regiões, a utilização do Mehlich-1 é controversa. O extrator sofre maior desgaste devido aos solos serem menos ácidos, no entanto, a solubilização de fosfatos de cálcio pode superestimar o P disponível (Freire, 2001), salvo na presença de plantas capazes de acidificar o solo da rizosfera, como as leguminosas (Novais \& Smyth, 1999).

A determinação da quantidade de nutrientes que se encontra no solo em forma disponível às plantas é um dos principais objetivos dos estudos de avaliação da fertilidade do solo. Porém, esse objetivo não pode ser atingido de maneira simples, devido à complexidade dos processos envolvidos na dinâmica dos nutrientes no sistema solo-soluçãoplanta (Cantarutti et al., 2007). Assim, um valor expresso num laudo de análise do solo pode ou não refletir a real disponibilidade de um nutriente (Silva et al., 2004), necessitando de experimentos de calibração. Desse modo, o teor ou nível crítico de um nutriente no solo corresponde ao valor de sua concentração que permite separar populações de solos com alta e baixa probabilidade de resposta às adições do nutriente avaliado (Alvarez V, 1996) e é variável entre solos e plantas e entre plantas e solos (Fonseca et al., 1997).
Assim, este trabalho objetivou (a) avaliar a disponibilidade de P por diferentes extratores químicos em solos com elevados teores de Ca e de reação neutra, comparativamente a solos hematíticos, e (b) correlacionar os teores críticos no solo e a adsorção de P em função do tempo de incubação.

\section{MATERIAL E MÉTODOS}

Este trabalho foi realizado em casa de vegetação no Centro de Pesquisa de Solos da Universidade Federal Rural de Pernambuco. Utilizaram-se amostras de dois solos, coletadas da camada subsuperficial, ocorrentes no Estado de Pernambuco: camada 30-56 cm, de um Nitossolo Vermelho distroférrico (NVdf), textura muito argilosa e predomínio de óxidos de $\mathrm{Fe}$ (hematita) proveniente da Zona da Mata; camada 5-25 cm, de um Vertissolo Ebânico órtico (VEo), textura argilosa e predomínio de minerais 2:1 (esmectita) proveniente do Sertão (Figura 1). Os solos das amostras foram secos ao ar, destorroados e passados em peneira de $2 \mathrm{~mm}$, tanto para a instalação do experimento como para as análises físicas e químicas realizadas conforme Embrapa (1997) (Tabela 1).

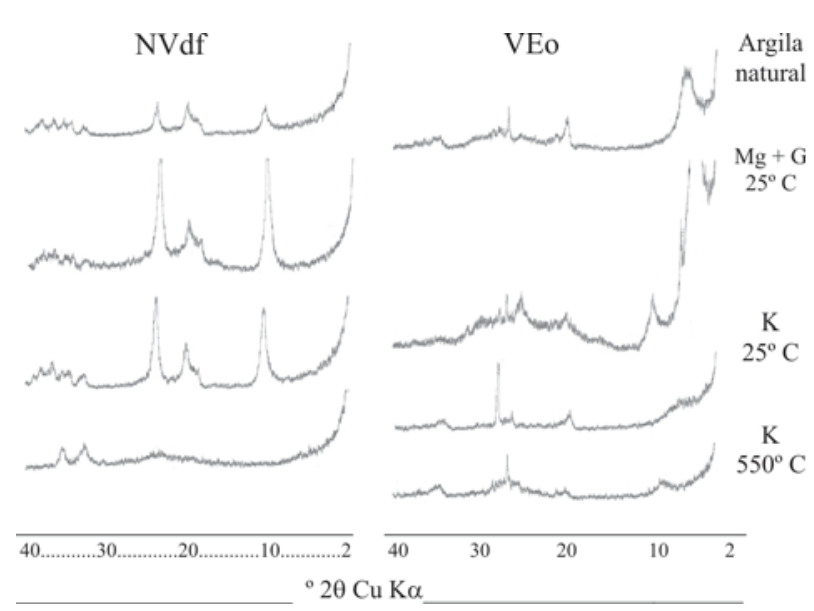

Figura 1. Difratogramas da fração argila dos solos com a presença de hematita no Nitossolo Vermelho distroférrico (NVdf) e argilominerais 2:1 no Vertissolo Ebânico órtico (VEo). 
Tabela 1 - Caracterização física e química dos solos Nitossolo Vermelho distroférrico (NVdf), proveniente da Zona da Mata, e Vertissolo Ebânico órtico (VEo), proveniente do Sertão, Pernambuco

\begin{tabular}{|c|c|c|}
\hline \multirow{2}{*}{ Característica } & \multicolumn{2}{|c|}{ Solo } \\
\hline & NVdf & VEo \\
\hline Areia $\left(\right.$ dag $\left.\mathrm{kg}^{-1}\right)$ & 11,8 & 29,8 \\
\hline Silte (dag kg-1) & 17,5 & 15,5 \\
\hline Argila $\left(\right.$ dag $\left.\mathrm{kg}^{-1}\right)$ & 70,7 & 54,7 \\
\hline Ds $\left(\mathrm{g} \mathrm{cm}^{-3}\right)^{(1)}$ & 1,12 & 1,72 \\
\hline $\mathrm{Dp}\left(\mathrm{g} \mathrm{cm}^{-3}\right)^{(2)}$ & 2,89 & 2,56 \\
\hline Cc $(\%)^{(3)}$ & 39,7 & 39,5 \\
\hline PMP $(\%)^{(4)}$ & 29,6 & 27,5 \\
\hline 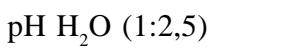 & 4,4 & 6,8 \\
\hline P Mehlich-1 (mg dm $\left.{ }^{-3}\right)$ & 2,4 & 495,6 \\
\hline P Mehlich-3 (mg dm-3) & 3,5 & 26,0 \\
\hline P Bray-1 (mg dm $\left.{ }^{-3}\right)$ & 0,4 & 15,4 \\
\hline $\mathrm{Na}\left(\mathrm{cmol}_{\mathrm{c}} \mathrm{dm}^{-3}\right)$ & 0,03 & 1,39 \\
\hline $\mathrm{K}\left(\mathrm{cmol}_{\mathrm{c}} \mathrm{dm}^{-3}\right)$ & 0,02 & 0,3 \\
\hline $\mathrm{Ca}+\mathrm{Mg}\left(\mathrm{cmol}_{\mathrm{c}} \mathrm{dm}^{-3}\right)$ & 1,2 & 72,7 \\
\hline $\mathrm{Al}\left(\mathrm{cmol}_{\mathrm{c}} \mathrm{dm}^{-3}\right)$ & 0,45 & 0,0 \\
\hline $\mathrm{H}+\mathrm{Al}\left(\mathrm{cmol}_{\mathrm{c}} \mathrm{dm}^{-3}\right)$ & 5,85 & 1,48 \\
\hline C org. $\left(\mathrm{g} \mathrm{kg}^{-1}\right)$ & 0,76 & 5,8 \\
\hline CMAP $\left(\mathrm{mg} \mathrm{cm}^{-3}\right)^{(5)}$ & 2,44 & 0,31 \\
\hline $\mathrm{EA}\left(\mathrm{mg} \mathrm{L}^{-1}\right)^{-1(6)}$ & 1,11 & 0,35 \\
\hline P-rem $\left(\mathrm{mg} \mathrm{L}^{-1}\right)^{(7)}$ & 0,37 & 37,3 \\
\hline
\end{tabular}

Para avaliar a capacidade máxima de adsorção de $\mathrm{P}$ (CMAP), amostras de $2,5 \mathrm{~cm}^{3}$ de terra fina seca ao ar (TFSA) de cada solo receberam 11 doses de $\mathrm{P}\left(\mathrm{KH}_{2} \mathrm{PO}_{4}\right)$, situadas no intervalo de 0 a $260 \mathrm{mg} \mathrm{L}^{-1}$ para o NVdf e de 0 a $80 \mathrm{mg} \mathrm{L}^{-1}$ para o VEo (Alvarez V et al., 2000). As doses foram definidas de acordo com o valor do fósforo remanescente (P-rem) de cada solo (Tabela 1), conforme estabelece Alvarez V et al. (2000). No NVdf as doses foram 0, 26, 52, 78, 104, 130, 156, 182, 208, 234 e $260 \mathrm{ml} \mathrm{L}^{-1}$ de P. No VEo foram 0, 8, 16, 24, 32, 40, 48, 56, 64, 72 e $80 \mathrm{ml} \mathrm{L}^{-1}$ de P. As doses foram aplicadas utilizando-se $25 \mathrm{~mL}$ de uma solução de equilíbrio $\left(\mathrm{CaCl}_{2} \cdot 2 \mathrm{H}_{2} \mathrm{O} 0,01 \mathrm{~mol} \mathrm{~L}^{-1}\right.$, contendo as diferentes doses de $\mathrm{P}$ ). A solução de equilíbrio teve a mesma concentração para todas as doses de P e é utilizada para manter a mesma força iônica nas diferentes soluções. O P-rem foi determinado de acordo com Alvarez $\mathrm{V}$ et al. (2000).

Amostras de $2 \mathrm{~kg}$ do NVdf receberam uma mistura de $\mathrm{CaCO}_{3}$ e $\mathrm{MgCO}_{3}$ numa relação molar de 4:1, por um período de 60 dias anterior à aplicação dos tratamentos, sendo a quantidade do corretivo estimada pela acidez potencial (H+Al) dos solos (Freire, 2001). No VEo não houve necessidade de correção da acidez.
As unidades experimentais consistiram de sacos plásticos contendo $2 \mathrm{~kg}$ de solo, que receberam as doses de $\mathrm{P}$ aplicadas por meio de soluções de $\mathrm{KH}_{2} \mathrm{PO}_{4}, \mathrm{NH}_{4} \mathrm{H}_{2} \mathrm{PO}_{4} \mathrm{e}$ $\mathrm{NaH}_{2} \mathrm{PO}_{4}$, correspondentes a 0, 20, 50, 80 e 100\% da CMAP. Utilizaram-se três fontes de $\mathrm{P}$ com o objetivo de balancear adequadamente o $\mathrm{K}$ e o $\mathrm{N}$ em todos os tratamentos. $\mathrm{O}$ uso de uma única fonte acarretaria quantidade excessiva de $\mathrm{K}$ ou N. O Na da fonte $\mathrm{NaH}_{2} \mathrm{PO}_{4}$ foi balanceado, assim como todos os demais nutrientes, com exceção do P. As amostras foram incubadas por 90, 60, 30, 15 e 0,5 dias. Os períodos de incubação foram iniciados em diferentes épocas, de modo a se ter, num mesmo e último dia, os diferentes períodos de equilíbrio solo-P satisfeitos. Durante todo período de incubação manteve-se a umidade em torno de $80 \%$ da sua capacidade de campo determinada pelo método da panela de pressão (Embrapa, 1997). Utilizou-se água destilada para repor as perdas por evaporação e manter a umidade adequada e previamente estabelecida. Os sacos plásticos eram pesados diariamente. No último dia de incubação, foram retiradas amostras de solo de cada saco plástico, de modo a obterem-se $100 \mathrm{~g}$ de solo seco. A secagem foi realizada a $60{ }^{\circ} \mathrm{C}$ em copos plásticos, por 48 horas, em estufa de circulação forçada de ar (Quaggio et al., 2001). Para cada tratamento, determinou-se o teor de $\mathrm{P}$ pelos extratores Mehlich-1 ( $\mathrm{HCl}$ 0,05 mol L-1 $+\mathrm{H}_{2} \mathrm{SO}_{4}$ 0,0125 mol L-1), Mehlich-3 ( $\mathrm{NH}_{4} \mathrm{~F}$ 0,015 $\mathrm{mol} \mathrm{L}^{-1}+\mathrm{CH}_{3} \mathrm{COOH} \mathrm{0,2} \mathrm{mol} \mathrm{L}^{-1}+$ $\mathrm{NH}_{4} \mathrm{NO}_{3} 0,25 \mathrm{~mol} \mathrm{~L}^{-1}+\mathrm{HNO}_{3}$ 0,013 $\mathrm{mol} \mathrm{L}^{-1}+$ EDTA 0,001

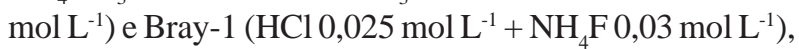
numa relação solo:extrator de $1: 10\left(4 \mathrm{~cm}^{3}\right.$ de TFSA e 40 $\mathrm{mL}$ da solução extratora). A avaliação de P no extrato foi realizada por colorimetria, de acordo com Braga \& Defelipo (1974).

Terminada a amostragem de todos os tratamentos, os sacos plásticos foram acondicionados em vasos plásticos de 2,5 L e levados para a casa de vegetação. Foram dispostos em um arranjo fatorial 2 x 5 x 5 (dois solos, cinco doses de $\mathrm{P}$ e cinco tempos de incubação), com os tratamentos distribuídos em blocos ao acaso, com três repetições.

Imediatamente semeou-se o milho cultivar BR 106, e aos 15 dias após o desbaste, mantendo-se duas plantas por vaso, realizou-se uma adubação com $60 ; 1,2 ; 0,8 ; 1,5$; 3,5; 0,15; e $4 \mathrm{mg} \mathrm{dm}^{-3}$ de S, Cu, B, Fe, Mn, Mo e Zn, respectivamente. Durante todo o período de cultivo, a umidade dos solos foi mantida a $80 \%$ da capacidade de campo (Embrapa, 1997) por meio da pesagem diária dos vasos com o objetivo de repor a água perdida por evapotranspiração.

A colheita da parte aérea foi realizada aos 35 dias após a semeadura. O material colhido foi seco em estufa a $60^{\circ} \mathrm{C}$ por 72 horas, sendo a massa da matéria seca pesada, triturada e mineralizada por uma mistura nítrico-perclórica (Bataglia et al., 1983), obtendo-se extratos nos quais foram determinados os teores de $\mathrm{P}$ por colorimetria (Braga \& Defelipo, 1974). 
Os dados foram submetidos à análise de variância, e quando o efeito dos tratamentos foi significativo, ajustaram-se equações polinomiais entre o teor de P extraído do solo e a produção de matéria seca como variáveis dependentes das doses aplicadas e dos tempos de incubação. A partir de modelos de regressão, foram estimadas doses para obtenção de $90 \%$ da produção máxima de massa de matéria seca da parte aérea. Com base nas doses estimadas e na regressão P extraído em função do aplicado, foram definidos os teores críticos nos solos. Correlacionouse o $\mathrm{P}$ extraído pelos extratores com o $\mathrm{P}$ absorvido e acumulado pelas plantas de milho para cada solo. O programa estatístico utilizado foi o SAEG (1999).

\section{RESULTADOS E DISCUSSÃO}

Observou-se que as menores taxas de recuperação do P foram obtidas para o NVdf (Tabela 2), condizente com seu baixo valor de P-rem e sua alta CMAP (Tabela 1). Especificamente no caso do VEo, o P extraído apresentou valores elevados (Tabela 2), provavelmente pelos altos valores de $\mathrm{P}$ ligado a Ca (P-Ca), em consequência dos altos teores de $\mathrm{Ca}+\mathrm{Mg}$ apresentados por esse solo (Tabela 1) e pela capacidade do Mehlich-1 ( $\mathrm{pH}$ 1,2) em solubilizar esses compostos.

O relacionamento entre a produção de massa de matéria seca e as doses de $\mathrm{P}$ aplicadas nos solos mostrou que, independentemente do tempo de incubação, o NVdf produziu mais massa de matéria seca do que o VEo (Tabela 3). A produção de massa de matéria seca da parte aérea do milho aumentou com o tempo de incubação no NVdf e manteve-se praticamente constante no VEo (Tabela 3). No NVdf, mesmo havendo diminuição no valor do P extraído por Mehlich-1, Mehlich-3 e Bray-1 (Tabela 2) com o aumento do tempo de incubação, houve aumento na produção de massa de matéria seca (Tabela 3), provavelmente pela maior eficiência na utilização do P absorvido. Essa eficiência nutricional pode ser calculada pela razão entre a produção de massa de matéria seca e o conteúdo de P. Segundo Novais \& Smyth (1999), as plantas se ajustam na utilização do P em solo com elevada CMAP.

As correlações entre o P extraído e o P absorvido pelo milho foram positivas e significativas para os três extra-

Tabela 2 - Equações de regressão ajustadas para os teores de fósforo no solo $\left(\mathrm{mg} \mathrm{dm}^{-3}\right.$ ) recuperados pelos extratores Mehlich-1, Mehlich-3 e Bray-1, como variável dependente das doses de fósforo aplicadas ( $\mathrm{mg} \mathrm{dm}^{-3}$ ) em diferentes tempos de incubação

\begin{tabular}{|c|c|c|c|c|}
\hline \multirow{2}{*}{$\begin{array}{l}\text { Tempo } \\
\text { (dias) }\end{array}$} & \multicolumn{4}{|c|}{ Solo } \\
\hline & \multicolumn{2}{|l|}{ NVdf } & VEo & \\
\hline & \multicolumn{4}{|c|}{ Mehlich-1 } \\
\hline 0,5 & $\hat{y}=-60,0019+0,329644 * * x$ & $\mathrm{R}^{2}=0,884$ & $\hat{y}=318,684+1,30294 * * x$ & $\mathrm{R}^{2}=0,930$ \\
\hline 15 & $\hat{y}=-62,1401+0,538923^{* *} x$ & $\mathrm{R}^{2}=0,890$ & $\hat{y}=573,539+0,738812^{* *} x$ & $\mathrm{R}^{2}=0,930$ \\
\hline 30 & $\hat{y}=-90,5948+0,405231^{* *} x$ & $\mathrm{R}^{2}=0,969$ & $\hat{y}=484,299+0,529157^{\otimes} x$ & $\mathrm{R}^{2}=0,418$ \\
\hline 60 & $\hat{y}=-64,0953+0,390145^{* *} x$ & $\mathrm{R}^{2}=0,960$ & $\hat{y}=480,095+0,337939^{\otimes} x$ & $\mathrm{R}^{2}=0,571$ \\
\hline \multirow[t]{2}{*}{90} & $\hat{y}=-106,594+0,330878^{* *} x$ & $\mathrm{R}^{2}=0,926$ & $\hat{y}=401,958+0,257352^{\otimes} x$ & $\mathrm{R}^{2}=0,422$ \\
\hline & \multicolumn{4}{|c|}{ Mehlich-3 } \\
\hline 0,5 & $\hat{y}=-59,2299+0,280801^{* *} x$ & $\mathrm{R}^{2}=0,936$ & $\hat{y}=-19,1555+1,56088^{* *} x$ & $\mathrm{R}^{2}=0,914$ \\
\hline 15 & $\hat{y}=-45,7229+0,269964^{* *} x$ & $\mathrm{R}^{2}=0,885$ & $\hat{y}=16,3171+0,562068^{* * *} x$ & $\mathrm{R}^{2}=0,993$ \\
\hline 30 & $\hat{y}=-61,4246+0,237040 * * x$ & $\mathrm{R}^{2}=0,951$ & $\hat{y}=23,5025+0,685503 * x$ & $\mathrm{R}^{2}=0,754$ \\
\hline 60 & $\hat{y}=-52,1349+0,240336^{* *} x$ & $\mathrm{R}^{2}=0,962$ & $\hat{y}=24,2023+0,340468^{*} * x$ & $\mathrm{R}^{2}=0,951$ \\
\hline \multirow[t]{2}{*}{90} & $\hat{y}=-82,5138+0,238515^{* *} x$ & $\mathrm{R}^{2}=0,911$ & $\hat{y}=26,7703+0,452378 * * * x$ & $\mathrm{R}^{2}=0,996$ \\
\hline & \multicolumn{4}{|c|}{ Bray-1 } \\
\hline 0,5 & $\hat{y}=-104,567+0,519683^{* *} x$ & $\mathrm{R}^{2}=0,937$ & $\hat{y}=-30,0398+1,82718^{*} x$ & $\mathrm{R}^{2}=0,920$ \\
\hline 15 & $\hat{y}=-81,2109+0,410593 * x$ & $\mathrm{R}^{2}=0,856$ & $\hat{y}=-4,74542+0,523834 * x$ & $\mathrm{R}^{2}=0,840$ \\
\hline 30 & $\hat{y}=-105,925+0,400045^{* * x}$ & $\mathrm{R}^{2}=0,958$ & $\hat{y}=18,1786+0,735904 * x$ & $\mathrm{R}^{2}=0,670$ \\
\hline 60 & $\hat{y}=-129,401+0,438119^{* * x}$ & $\mathrm{R}^{2}=0,916$ & $\hat{y}=23,1548+0,451280^{* *} x$ & $\mathrm{R}^{2}=0,956$ \\
\hline 90 & $\hat{y}=-130,426+0,351076^{* *} x$ & $\mathrm{R}^{2}=0,905$ & $\hat{y}=14,6853+0,474977 * * * x$ & $\mathrm{R}^{2}=0,999$ \\
\hline
\end{tabular}

***,**,*, e ${ }^{\otimes}$ significativo a 0,$1 ; 1 ; 5 ;$ e $10 \%$ de probabilidade, respectivamente. 
Tabela 3 - Equações de regressão ajustadas para produção de matéria seca de milho (g/vaso) como variável dependente das doses de fósforo aplicadas $\left(\mathrm{mg} \mathrm{dm}^{-3}\right)$ nos diferentes tempos de incubação

\begin{tabular}{|c|c|c|c|}
\hline Solo & Tempo (dias) & Equações & $\mathbf{R}^{2}$ \\
\hline \multirow{5}{*}{ NVdf } & 0,5 & $\hat{\mathrm{y}}=0,0482273+0,842514 * \sqrt{x}-0,0117178^{*} \mathrm{x}$ & 0,975 \\
\hline & 15 & $\hat{\mathrm{y}}=0,278724+1,31281 * \sqrt{x}-0,0206760 * \mathrm{x}$ & 0,946 \\
\hline & 30 & $\hat{\mathrm{y}}=0,272156+1,50182 * * \sqrt{x}-0,0218980 * \mathrm{x}$ & 0,984 \\
\hline & 60 & $\hat{\mathrm{y}}=-0,360764+1,17116 * \sqrt{x}-0,0130313 * \mathrm{x}$ & 0,974 \\
\hline & 90 & $\hat{\mathrm{y}}=0,326052+1,00556 * \sqrt{x}-0,0101482 * \mathrm{x}$ & 0,982 \\
\hline \multirow{5}{*}{ VEo } & 0,5 & $\hat{y}=3,78661+0,131233 * x-0,000377277 * x^{2}$ & 0,927 \\
\hline & 15 & $\hat{\mathrm{y}}=5,40402+1,35663^{*} \sqrt{x}-0,0503847^{\otimes} \mathrm{x}$ & 0,894 \\
\hline & 30 & $\hat{y}=5,36449+0,842577^{\otimes} \sqrt{x}-0,0325416^{\mathrm{ns}} \mathrm{x}$ & 0,712 \\
\hline & 60 & $\hat{y}=5,37314+0,0701190 * x-0,000182315^{*} x^{2}$ & 0,953 \\
\hline & 90 & $\hat{\mathrm{y}}=3,2419+1,48516^{\otimes} \sqrt{x}-0,0597997^{\mathrm{ns}} \mathrm{x}$ & 0,822 \\
\hline
\end{tabular}

**,*, e ${ }^{\otimes}$ significativo a 1,5 e $10 \%$ de probabilidade, respectivamente e ${ }^{\text {ns }}$ não significativo.

tores testados e para os dois solos (Tabela 4). No NVdf o extrator Mehlich-1 apresentou o maior coeficiente e significância da correlação ( $\mathrm{r}=0,9906 * * *)$, mostrando sua eficiência na predição do P disponível em solos hematíticos

Tabela 4. Coeficiente de correlação linear simples entre o P absorvido e o acumulado pelas plantas de milho com o P extraído pelos extratores Mehlich-1, Mehlich-3, Bray-1

\begin{tabular}{|c|c|}
\hline $\begin{array}{l}\text { Fósforo extraído } \\
\qquad\left(\mathrm{mg} \mathrm{dm}^{-3}\right)\end{array}$ & $\begin{array}{c}\text { Fósforo absorvido e acumulado } \\
\text { (mg/vaso) }\end{array}$ \\
\hline \multicolumn{2}{|r|}{ NVdf } \\
\hline Mehlich-1 & $0,9906 * * *$ \\
\hline Mehlich-3 & $0,9846 * *$ \\
\hline Bray-1 & $0,9834 * *$ \\
\hline \multicolumn{2}{|r|}{ VEo } \\
\hline Mehlich-1 & $0,7839^{\otimes}$ \\
\hline Mehlich-3 & 0,9015* \\
\hline Bray-1 & 0,9109* \\
\hline
\end{tabular}

em que predominam formas de $\mathrm{P}$ ligado a $\mathrm{Fe}$ (P-Fe). No entanto, no VEo a capacidade de predição do $\mathrm{P}$ disponível pelo Mehlich-1 diminuiu significativamente ( $\mathrm{r}=$ $\left.0,7839^{\otimes}\right)$, mostrando que esse extrator, pelos baixos valores de $\mathrm{pH}$, responsáveis pela maior solubilização do $\mathrm{P}$ ligado a $\mathrm{Ca}$ (P-Ca), não foi adequado a solos ricos em $\mathrm{Ca}$ e, ou, para avaliar solos tratados com fosfato natural (Braga et al., 1991; Silva \& Raij, 1999).

Correlacionando-se os extratores, constatou-se significativo e elevado coeficiente de correlação entre o Mehlich-3 e Bray-1 ( $r=0,977^{* * *}$ ), o que permite recomendar um ou outro como extrator do P disponível. No entanto, pela praticidade e baixo custo do Bray-1, sugere-se a utilização deste extrator como referencial para futuras pesquisas com extratores para predição do P disponível em solos com predominância de Ca, inclusive com a utilização do Olsen, apesar de sua pequena solubilidade, o que dificulta sua operacionalização em laboratórios de rotina.

O teor crítico de P no NVdf foi, em média, menor do que no VEo (Tabela 5) pelo menor valor de P-rem e pela

Tabela 5 - Níveis críticos de fósforo nos solos pelos extratores Mehlich-1, Mehlich-3, Bray-1 nos diferentes tempos de incubação

\begin{tabular}{|c|c|c|c|c|c|c|}
\hline \multirow{3}{*}{ Solo } & \multirow{3}{*}{ Extrator } & \multicolumn{4}{|c|}{ Níveis críticos de fósforo no solo $\left(\mathrm{mg} \mathrm{dm}^{-3}\right)$} & \\
\hline & & \multicolumn{5}{|c|}{ Tempo de incubação (dias) } \\
\hline & & 0,5 & 15 & 30 & 60 & 90 \\
\hline \multirow{3}{*}{ NVdf } & Mehlich-1 & 140 & 190 & 130 & 307 & 271 \\
\hline & Mehlich-3 & 111 & 81 & 68 & 176 & 190 \\
\hline & Bray-1 & 211 & 111 & 112 & 366 & 271 \\
\hline \multirow{3}{*}{ VEo } & Mehlich-1 & 467,60 & 620,80 & 513,00 & 517,30 & 417,70 \\
\hline & Mehlich-3 & 159,25 & 52,30 & 60,50 & 61,65 & 54,40 \\
\hline & Bray-1 & 178,80 & 28,80 & 58,00 & 72,80 & 43,70 \\
\hline
\end{tabular}


elevada CMAP daquele solo (Tabela 1), corroborando com outros pesquisadores (Bahia Filho et al., 1982; Novais \& Smyth, 1999; Silva et al., 2004). Os teores críticos de P no NVdf foram decrescentes com o aumento do tempo de incubação. Entretanto, a partir dos 60 dias esses níveis críticos se elevaram (Tabela 5). É provável que a partir daí, os sítios de adsorção tenham se saturado, fazendo com que o NVdf apresentasse comportamento semelhante a solos arenosos. Loganathan \& Fernando (1980) e Singh et al. (1983) mostraram que quando se aplica uma fonte solúvel de $\mathrm{P}$ num solo, frequentemente grande parte do aplicado é adsorvido rapidamente, com consequente saturação dos sítios de adsorção. Segundo Gonçalves et al. (1985), a velocidade de adsorção é maior em solos com maior CMAP, e quanto maior a saturação dos sítios de adsorção menor a velocidade de transformação de P lábil em P não lábil.

\section{CONCLUSÕES}

A capacidade de predição do P disponível pelo método de Mehlich-1 não é adequada para solos com predomínio de argilominerais 2:1 ricos em Ca, como o Vertissolo Ebânico órtico.

Os teores críticos de P no solo para o milho apresentam-se decrescentes com o aumento do tempo de incubação, independentemente dos solos.

\section{REFERÊNCIAS}

Alvarez V VH (1996) Correlação e calibração de métodos de análises de solo. In: Alvarez V VH, Fontes LEF \& Fontes MPF (Eds.) O solo nos grandes domínios morfoclimáticos do Brasil e o desenvolvimento sustentado. Viçosa, Sociedade Brasileira de Ciência do Solo. p.615-646.

Alvarez V VH, Novais RF, Dias LE \& Oliveira JA (2000) Determinação e uso do fósforo remanescente. Viçosa, Sociedade Brasileira de Ciência do Solo. p.27-32 (Boletim Informativo, 25).

Bahia Filho AFC, Vasconcenlos CA, Santos HL, Mendes JP, Pita GVE \& Oliveira AC (1982) Formas de fósforo inorgânico e fósforo “disponível” em um Latossolo Vermelho-Escuro, fertilizado com diferentes fosfatos. Revista Brasileira de Ciência do Solo, 6:99-104.

Bataglia OC, Furlani AMC, Teixeira JPF, Furlani PR \& Gallo JR (1983) Métodos de análise química de plantas. Campinas, Instituto Agronômico. 20p. (Boletim técnico, 78).

Braga JM \& Defelipo BV (1974) Determinação espectrofotométrica de fósforo em extratos de solo e material vegetal. Revista Ceres, 21:73-85.

Braga NR, Mascarenhas HAA, Bulisani EA, Raij B van, Feitosa CT \& Hirose R (1991) Eficiência agronômica de nove fosfatos em quatro cultivos consecutivos de soja. Revista Brasileira de Ciência do Solo, 15:315-319.

Cantarutti RB, Barros NF, Martinez HEP \& Novais RF (2007) Avaliação da fertilidade do solo e recomendação de fertilizantes. In: Novais RF, Alvarez V VH, Barros NF, Fontes RLF, Cantarutti RB \& Neves JCL (Eds.) Fertilidade do solo. Viçosa, Sociedade Brasileira de Ciência do Solo. p.769-850.

EMBRAPA (1997) Manual de métodos de análise do solo. Rio de Janeiro, CNPS. 212p.
Fonseca DM, Gomide JA, Alvarez V VH \& Novais RF (1997) Fatores que influenciam os níveis críticos de fósforo para o estabelecimento de gramíneas forrageiras: I. Casa de vegetação. Revista Brasileira de Ciência do Solo, 21:27-34.

Freire FJ (2001) Sistema para cálculo do balanço nutricional e recomendação de corretivos e fertilizantes para cana-de-áçucar. Tese de Doutorado. Viçosa, Universidade Federal de Viçosa. 144p.

Gonçalves JLM, Firme DJ, Novais RF \& Ribeiro AC (1985) Cinética de adsorção de fósforo em solos de cerrado. Revista Brasileira de Ciência do Solo, 9:107-111.

Loganathan P \& Fernando WT (1980) Phosphorus sorption by some coconut-growing acid soils of Sri Lanka and its relationship to selected soil properties. Journal Science Food Agriculture, 31:709-717.

Mesquita Filho MV \& Torrent J (1993) Phosphate sorption as related to mineralogy of a hydrosequence of soils from the Cerrado region (Brazil). Geoderma, 58:107-123.

Novais RF \& Smyth TJ (1999) Fósforo em solo e planta em condições tropicais. Viçosa, UFV. 399p.

Quaggio JA, Cantarella H, Abreu CA \& Raij BV (2001) Registro e preparo de amostras. In: Raij BV, Andrade JC, Cantarella H \& Quaggio JA (Eds.) Análise química para avaliação da fertilidade de solos tropicais. Campinas, Instituto Agronômico. p.136-141.

SAEG (1999) Sistema para Análises Estatísticas. Viçosa, Fundação Arthur Bernardes. CD-ROM.

Silva EMB, Freire FJ, Santos MVF, Silva TJA \& Freire MBGS (2004) Níveis críticos de fósforo para Brachiaria brizantha e suas relações com características físicas e químicas em solos de Pernambuco. Revista Brasileira da Ciência do Solo, 28:281288.

Silva FC \& Raij B van (1999) Disponibilidade de fósforo em solos avaliada por diferentes extratores. Pesquisa Agropecuária Brasileira, 34:267-288.

Singh R, Möller MRF \& Ferreira WA (1983) Cinética da sorção de fósforo em solos dos trópicos úmidos da Amazônia. Revista Brasileira de Ciência do Solo, 7:227-231. 